\title{
Activity Support for Seniors Using Public Displays: A Proof of Concept
}

\author{
Julian Fietkau \\ Laura Stojko \\ julian.fietkau@unibw.de \\ laura.stojko@unibw.de \\ Universität der Bundeswehr München \\ Neubiberg, Germany
}

\begin{abstract}
Seniors face many challenges in their daily activities regarding mobility and accessibility. We have designed and prototyped a system of networked public displays to support them, particularly regarding outdoor pedestrian navigation. This article describes the process and results of a qualitative evaluation of this prototype system, which was conducted with seven participants, a mixture of older adults and experts on geriatric care. Based on insights gained from these interviews, we provide guidance on the design of outdoor activity support systems for seniors.
\end{abstract}

\section{CCS CONCEPTS}

- Human-centered computing $\rightarrow$ Displays and imagers; Empirical studies in ubiquitous and mobile computing; • Social and professional topics $\rightarrow$ Seniors.

\section{KEYWORDS}

human-computer interaction, public displays, activity support, navigation assistance, seniors

ACM Reference Format:

Julian Fietkau and Laura Stojko. 2021. Activity Support for Seniors Using Public Displays: A Proof of Concept. In Mensch und Computer 2021 (MuC '21), September 5-8, 2021, Ingolstadt, Germany. ACM, New York, NY, USA, 5 pages. https://doi.org/10.1145/3473856.3474002

\section{INTRODUCTION}

With sensory and mobility limitations becoming more frequent in old age, many seniors face steadily increasing challenges when it comes to navigating through the urban environment and conducting their daily activities. In a survey of over 2.000 people aged 65 and up that was conducted in the scope of this project [15], reasons such as unsafe pedestrian paths $(38 \%)$ or a lack of awareness about senior-accessible activities (21\%) are moderately often cited as factors that prevent older adults from participating in outside activities [19]. Related research $[12,18]$ substantiates unsafe pedestrian paths as a factor for seniors' reduced outdoor activity.

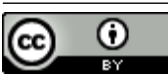

This work is licensed under a Creative Commons Attribution International 4.0 License.

MuC '21, September 5-8, 2021, Ingolstadt, Germany

(c) 2021 Copyright held by the owner/author(s).

ACM ISBN 978-1-4503-8645-6/21/09.

https://doi.org/10.1145/3473856.3474002
To tackle the challenges of accessing information about available activities, selecting them, and navigating to and from those activities, we have designed and prototyped an interactive system consisting of large and small public displays and central backend services to facilitate personalized assistance throughout the experience. The aim of the system is to motivate seniors to engage in outdoor activities and support them during the execution by increasing their subjective safety.

This paper describes the outcome of our qualitative evaluation to answer the central research question: How must large and small public displays be designed to support and increase the subjective safety of seniors during outdoor activities?

\section{RELATED WORK}

Within the broader conceptual space of supporting seniors in their everyday activities using interactive technology, a number of research projects and ideas have been proposed. For example, Krieg et al. [10] describe how personal mobility devices (such as wheelchairs) could be augmented with digital navigation assistance. Similarly, Ahmetovic et al. [1] and the m4guide project by Fraunhofer FOKUS [6] use smartphone apps to provide personalized navigation assistance to people with disabilities. The central difference between these approaches and ours is that we propose public devices with fixed installations in the urban space instead of personal devices. Kempter et al. [7] and Richter et al. [13] propose systems based around interactions with publically installed devices, but focus on indoor navigation.

Moving away from the focus on older users, there exists a slightly larger body of work on using public displays to support pedestrian navigation in general. However, most of them combine interactions with public and personal devices in some fashion. For example, Rukzio et al. [14] combine a public directional display with vibration signals emitted by a personal device in the user's pocket. Müller et al. [11] center the navigation on the personal mobile device, but augments it using sparse public displays. Kray et al. [9] avoid the use of personal devices by eschewing personalization completely, using public displays to provide non-personalized dynamic navigation assistance. Based on our literature review, we believe that our approach of publically installed devices for activity support and personalized navigation assistance for seniors is the first of its kind.

The concept and the individual parts of our system have been discussed in prior publications, specifically about the requirements analysis and the technical architecture [5], the persuasive design aspect anchored in the gamification research landscape [4], and more detailed guidance on the interaction design of small [16] and 
large public displays [8] as relevant to this kind of design. The following section nonetheless gives a short design overview.

\section{SYSTEM DESIGN}

As briefly introduced in Section 1, in terms of interactive hardware our system consists of sparsely deployed large interactive screens for activity selection, as well as a large number of small displays for navigation and activity support. These displays are connected with each other and with backend services for profile storage, activity management, and routing. Primary design considerations were that the system should be inclusive towards users with age-related impairments and that all functionality should be accessible without having to interact with a personal mobile device, since older users in particular often have difficulties with small touch screens regarding both reading and pointing.

Our devices can recognize registered seniors via Bluetooth when they approach. (Users who do not routinely carry a Bluetoothcapable personal mobile device can take advantage of this function by carrying a non-interactive $\mathrm{BLE}^{1}$ beacon in their pockets.) This enables personalized activity support. Users may instead also decide to use the large displays anonymously to learn about possible activities in their surroundings and forgo personalized activity support.

- Large interactive displays advertise possible activities. After recognizing a nearby user they can display a "personal activity board" where interesting activities can be added via drag-and-drop (Fig. 1). This activates the activity support during the execution. The personal activity board is designed to mirror the kind of pinboard people might have at home, where they would keep concert tickets, shopping lists, and other reminders.

- Our small public displays show helpful symbols (mainly arrows and squares) in personalized colors. The devices have a low-resolution display with a size of $3 \times 3 \mathrm{~cm}(1.2 \times 1.2$ inches $)$. An example of an arrow is displayed in Fig. 2. They are installed along footpaths and at intersections to provide realtime personalized navigation assistance, taking the safety and accessibility of specific pedestrian paths into account.

One of the main design decisions behind the presentation and selection of activities at our large information screen was to model them after the quest format commonly found in online games [2], in which players receive rewards for completing specific challenges, in order to increase motivation and lean into established patterns for gamified experiences. For more details on this aspect of the system, see Fietkau [4]. Among other details, this entailed specifying small material rewards for each activity as an added incentive. In general we would expect this to be a low-value extrinsic reward that fits the activity in question. We surmised from anecdotal experience that older participants may be particularly receptive to activities if they receive a concrete advantage for taking part, and the literature supports this to an extent [3].

${ }^{1}$ Bluetooth Low Energy - a standard for low-bandwidth Bluetooth communication allowing for very long battery life.

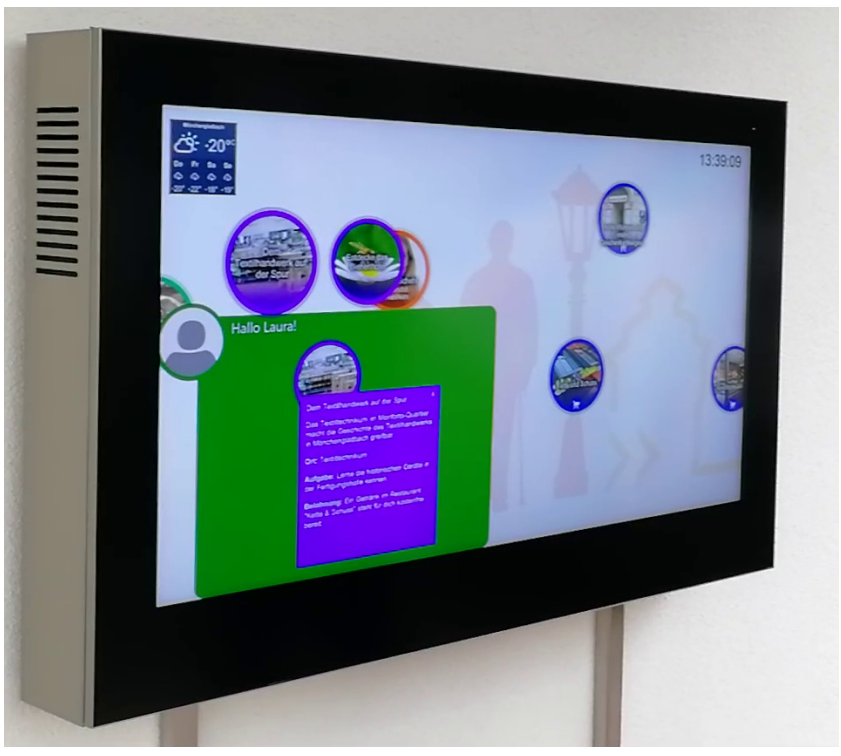

Figure 1: Large information screen at the Scooter-Park with a display of the personal activity board.

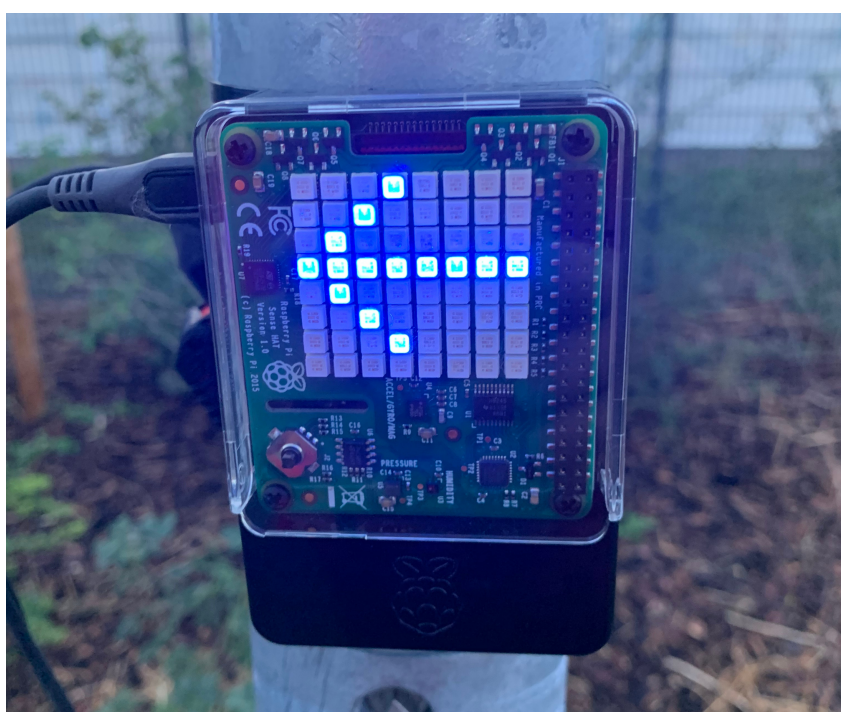

Figure 2: Small public display showing an arrow at the Scooter-Park.

\section{METHODOLOGY}

The initial plan for the activity support systems' evaluation consisted of a three-tiered long-term user study which had to be scrapped due to safety concerns, especially for seniors, during the COVID-19 pandemic. We decided to compress as much of our research as possible into a single study with a smaller number of participants, which happened in an enclosed area without random passers-by to minimize the risk of infection. 
We conducted the evaluation at the beginning of September 2020 in Mönchengladbach, Germany. The "Senioren-Scooter-Park" owned by our project partner Sozial-Holding Mönchengladbach $\mathrm{GmbH}$ was our evaluation location. The park is a training area intended for older adults to practice with various mobility aids on simulated streets and pathways without any danger from real traffic.

Participants were recruited by and through Sozial-Holding GmbH, our local connection to senior homes and assisted living programmes. Our evaluation timeframe was limited to one week and we planned for about 10-12 participants. For organizational reasons outside of our control, we received 9 confirmations in total, but due to bad weather during the week only 7 volunteers were able to participate in the evaluation. Among them were two seniors (aged 75 and 77) speaking from their own experience as older adults, and five employees or associates of the Sozial-Holding (aged 26, 40, 51, 61 and 64) who were asked to judge the system based on their geriatric care expertise and, depending on their age, also on their personal experiences. Four out of seven participants were female and all were from the area around Mönchengladbach. The two seniors live independently, one of them mentioned severe limitations regarding personal mobility. The experts reported that the senior home residents suffer from many physical and cognitive limitations. The two seniors reported doing outdoor activities more than twice a week (going to church, concerts, shopping, etc.). In contrast, our experts reported that the senior home residents rarely engage in outdoor activities, mainly only grocery shopping, doctor's appointments, or other events. In terms of technology use, seniors indicated that they use smartphones and computers frequently, but have no experience with large public displays.

At the beginning, users were asked to choose their favorite color which was then used for personalized information on the public displays (personal activity board and navigation symbols). The activity that was part of our scenario (a visit to the "bee hotel" at the southern edge of the park) appeared as a recommendation on the large public display screen. Participants were asked to interact with the screen to gather information about the activity and drag it onto their personal activity board to activate it. The small public displays placed along the way showed arrows and squares as navigational support. Upon completing the activity, participants received a flower as a quest reward to match the bee hotel theme. Fig. 3 displays an overview of the installation, including the positions of all public displays and the goal.

Each user session was structured in five parts:

(1) A pre-interview for general questions about physical limitations, technology affinity and experience, and motivation for outdoor activities.

(2) A user test at the large screen to find and activate the activity for the example scenario.

(3) A short post-interview to evaluate the interaction with the large screen.

(4) A walk through the Scooter-Park to the bee hotel following small public displays and back to the large display.

(5) A post-interview about the walk and usage of small public displays.

\footnotetext{
${ }^{2}$ https://www.sozial-holding.de/senioren-scooter-sharing.html
}

In all, we recorded 3 hours and 46 minutes of audio. In accordance with our participant privacy agreement, the audio recordings were destroyed after the transcription was complete. Each interview segment was semi-structured. We had a list of questions we wanted to cover, but we also attempted to get our participants to talk freely about their thoughts and impressions in order to gain insights we might not have anticipated. The total duration of the experiment varied between 21 and 45 minutes.

We evaluated the interview data following the qualitative data analysis process described by Strauss \& Corbin [17], which consists of open coding, axial coding and selective coding. This approach was chosen for its suitability for the analysis of interview transcripts, particularly since it prevents experimenter biases and preconceptions from clouding a clear view of the data. All interviews were analyzed in-depth in this fashion and central results are summarized below.

\section{RESULTS}

This section describes findings regarding activity selection at the large public display and activity support using small public displays.

\subsection{Activity Selection and Activation}

Regarding the quest framework and material rewards, our participants were split in two groups: a few were immediately taken by the idea, noting that extrinsic rewards would be a strong motivator for them, others were more put off by the externalization of motivation, preferring to decide by intrinsic factors and mentioning a preference for symbolic rewards over incentives of material value. All participants agreed, however, that the presentation format for activities (title, short description, dates and locations, accompanying photo) was very convenient and useful, especially for seniors.

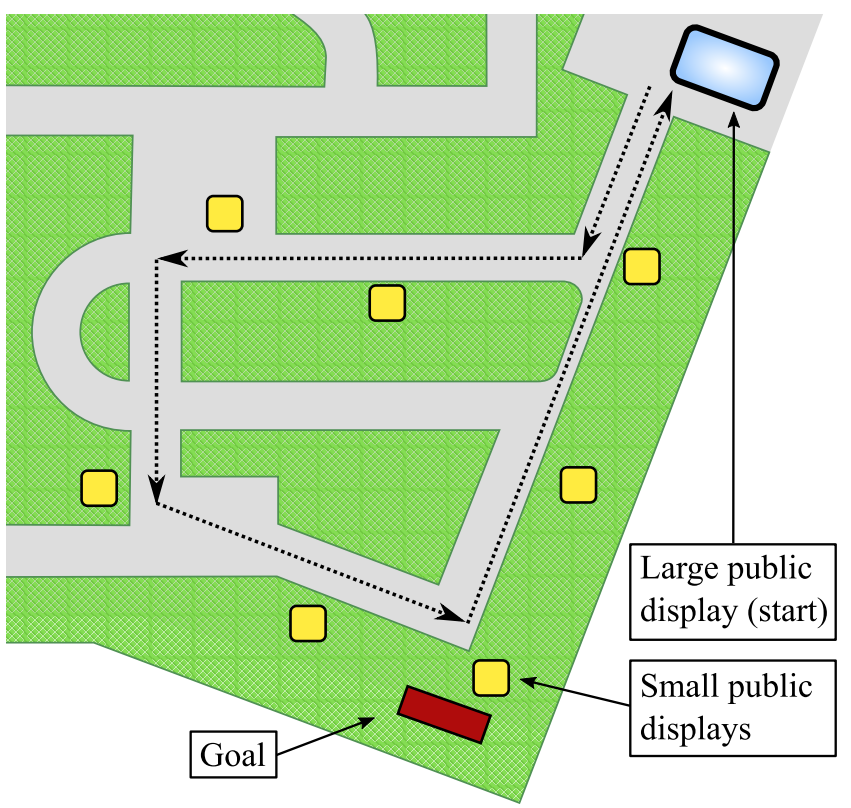

Figure 3: Map of all public displays installed at the ScooterPark for the evaluation. 
The participants determined the quest format to be a good motivator in the general sense. One of our design goals was to develop a system that could increase the awareness and informedness in the target user group regarding available activities. All participants agreed that they, and other people they know, would likely be better informed in this regard if our system were available in practice. In a few cases, participants even stated that they could see how the system could contribute to a societal shift towards more active and interwoven urban neighborhoods.

Nevertheless, the participants also mentioned some ideas that could have improved their experience during activity selection. One idea was to include the expected duration for a specific activity, which would help older adults to identify whether they are capable of completing it. Adding this information in a way that is highly useful for users with specific mobility needs would require consideration of the user's means of transportation (wheelchair, walker, un-aided walking, etc.) as the required time may differ significantly. Ideally, the system may take prior knowledge about a user's walking speed (from previous activities) into account. The background services in our system are theoretically capable of handling a rich user model that (provided the user consents to this) could use existing data on health and mobility to personalize the selection and display of activites. However, these functions were not used in the evaluation.

\subsection{Navigation and Activity Support}

The evaluation of the small public displays focussed on the overall activity support experience and wether the symbols were helpful and clear for navigating seniors during outdoor activities.

The participants emphasized the lightweight interaction with the small public displays as they were recognized via Bluetooth without any additional required steps. In order to ensure assistance during the whole activity it is necessary to evaluate the respective area and identify critical path points. Especially on these, displays are required. We had a few intersections where no screen was placed or it was not at the best location for an intersection (a few meters sideways). The participants criticized the positioning and lack of clear information for navigation at those areas. If older adults rely on guidance by small public displays, it is important to ensure a seamless coverage during the activity.

Overall, the participants experienced a higher degree of orientation and subjective safety by looking for the displays and consequently, higher awareness of their surroundings. Furthermore, the experience had a fun factor as it was like a treasure hunt: "What will be the next symbol?" "What could this symbol mean?"

Regarding the design, interpretation, and color we summarize the following insights: We used arrows and squares as navigation symbols on the path. Halfway, we positioned a screen that displayed a check mark and the goal "bee hotel" was represented by yellow stripes. All symbols started blinking as soon as they detected an approaching participant and stopped after they had moved out of the detection radius. Overall, the participants identified the different symbols and the greatest added value was provided by arrows, due to easy interpretation and understanding as they are commonly used in public. One issue was using arrows at intersections or for displaying a turn. The feedback regarding the check mark was overall positive as it was affirmative, some example reactions: "Well done!" "It's done!" In comparison to squares, which were criticized as unclear, the check mark was understood much better.

The outcome regarding the symbol's size was not clear, some criticized that they were too small. Nevertheless, their visibility was rated as good, especially due to their dynamic display (blinking). The public displays showed symbols in the previously selected personal color. This was used to personalize the information and get people to recognize the symbols. Most participants were able to identify the color and meaning: "This is my route." "If it is yellow, it is for me." Solely participants with green and red symbols firstly identified them as signal colors (green = good, well done and red = caution, danger, stop) rather than their personal color. Consequently, colors with a specific cultural meaning (e.g. signal color) should not be naively used for personalized navigation. In general, personalizing visual cues (by color or otherwise) becomes much more relevant when the system is used by multiple seniors simultaneously, which was not part of this evaluation, but was an important use case in the design process.

\section{CONCLUSION}

As a result of our qualitative evaluation, we identified that our participants felt motivated and safer with personalized activity support at the selection and while conducting the outdoor activity. The evaluation, even though it was conducted outside, has aspects of a laboratory experiment and not just a true field test. As such, any practical conclusions drawn by our participants are self-reported extrapolations based on the hypothetical scenario. To gain more clarity about the system's potential impact, "true" field tests in actual public contexts would be advantageous. Our evaluation was additionally curtailed by the COVID-19 pandemic. At the time of writing, it is extremely difficult to conduct practical evaluations with older users, especially field tests. We were externally limited not only in our study approach, but also in the number of participants. Nonetheless, we were able to gather and summarize some valuable qualitative insights into the design of public displays to increase seniors' sense of safety through navigation and activity support.

Apart from the obvious extrapolation to a larger evaluation with more participants, the multi-user and social aspects of our system cover important research questions (e.g. Do seniors feel exposed when using activity support? How to support activities of multiusers?) that could be investigated in the future. Another remaining topic is the navigation support at intersections. We identified issues by using simple arrows, further research needs to be done to identify better signals for a turn.

\section{ACKNOWLEDGMENTS}

This work has been supported by the Federal Ministry of Education and Research, Germany, under grant 16SV7443. We thank all project partners for their commitment. We particularly offer our thanks to Sozial-Holding Mönchengladbach GmbH, especially Helmut and Susanne Wallrafen as well as Jana Volk, for facilitating the location, recruiting participants, and working with us on a pandemic safety concept. 


\section{REFERENCES}

[1] Dragan Ahmetovic, Cole Gleason, Chengxiong Ruan, Kris Kitani, Hironobu Takagi, and Chieko Asakawa. 2016. NavCog: A Navigational Cognitive Assistant for the Blind. In Proceedings of the 18th International Conference on HumanComputer Interaction with Mobile Devices and Services (Florence, Italy) (MobileHCI '16). Association for Computing Machinery, New York, NY, USA, 90-99. https: //doi.org/10.1145/2935334.2935361

[2] Staffan Björk. 2018. Gameplay Design Patterns Collection: Quests. http://virt10. itu.chalmers.se/index.php?title $=$ Quests\&oldid $=26294$

[3] Rick Ferguson and Kelly Hlavinka. 2008. SegmentTalk: the difference engine: a comparison of loyalty marketing perceptions among specific US consumer segments. Fournal of Consumer Marketing 25 (March 2008). https://doi.org/10. $1108 / 07363760810858855$

[4] Julian Fietkau. 2019. Quests als Gestaltungsmittel zur Motivation und Struktur außerhäuslicher Aktivitäten für Senioren. In Mensch und Computer $2019-$ Workshopband (Hamburg, Germany), Florian Alt, Andreas Bulling, and Tanja Döring (Eds.). Gesellschaft für Informatik e.V., Bonn, Germany, 63-69. https: //doi.org/10.18420/muc2019-ws-591

[5] Julian Fietkau and Laura Stojko. 2020. A system design to support outside activities of older adults using smart urban objects. In Proceedings of the 18th European Conference on Computer-Supported Cooperative Work. EUSSET, Siegen, Germany. https://doi.org/10.18420/ecscw2020_ep07

[6] Fraunhofer FOKUS, ASCT. 2015. m4guide: Mobile multi-modal mobility guide Research project. https://www.fokus.fraunhofer.de/en/asct/m4guide

[7] Guido Kempter, Walter Ritter, and Andreas Künz. 2014. Guiding Light for the Mobility Support of Seniors. In Ambient Assisted Living, Reiner Wichert and Helmut Klausing (Eds.). Springer Berlin Heidelberg, Berlin, Heidelberg, 35-45.

[8] Michael Koch, Anna Kötteritzsch, and Julian Fietkau. 2017. Information Radiators - Using large screens and small devices to support awareness in urban space. In Proceedings of the International Conference on Web Intelligence 2017 (Leipzig, Germany) (WI '17), Amit P. Sheth, Axel Ngonga, Yin Wang, Elizabeth Chang, Dominik Slezak, Bogdan Franczyk, Rainer Alt, Xiaohui Tao, and Rainer Unland (Eds.). Association for Computing Machinery, New York, NY, USA, 1080-1084. https://doi.org/10.1145/3106426.3109039

[9] Christian Kray, Gerd Kortuem, and Antonio Krüger. 2005. Adaptive Navigation Support with Public Displays. In Proceedings of the 10th International Conference on Intelligent User Interfaces (San Diego, California, USA) (IUI '05). Association for Computing Machinery, New York, NY, USA, 326-328. https://doi.org/10. 1145/1040830.1040916

[10] Bernd Krieg-Brückner, Christian Mandel, Christoph Budelmann, Bernd Gersdorf and Antonio B. Martínez. 2015. Indoor and Outdoor Mobility Assistance. Springer International Publishing, Cham, 33-52. https://doi.org/10.1007/978-3-319-11866$6 \_3$

[11] Jörg Müller, Marc Jentsch, Christian Kray, and Antonio Krüger. 2008. Exploring Factors That Influence the Combined Use of Mobile Devices and Public Displays for Pedestrian Navigation. In Proceedings of the 5th Nordic Conference on HumanComputer Interaction: Building Bridges (Lund, Sweden) (NordiCHI '08). Association for Computing Machinery, New York, NY, USA, 308-317. https://doi.org/10 $1145 / 1463160.1463194$

[12] Charles Musselwhite. 2017. Creating a Convivial Public Realm for an Ageing Population. Being a Pedestrian and the Built Environment. Transport, Travel and Later Life 10 (Jan. 2017), 129-137. https://doi.org/10.1108/S2044-994120170000010005

[13] Julia Richter, Jeanine Lorenz, Maria Costantino, Verena Traubinger, Nico Tauchmann, Thomas Graichen, and Ulrich Heinkel. 2020. Dynamic Indoor Navigation and Orientation System for People with Impairments. In Proceedings of the Conference on Mensch Und Computer. Association for Computing Machinery, New York, NY, USA, 473-477. https://doi.org/10.1145/3404983.3410000

[14] Enrico Rukzio, Michael Müller, and Robert Hardy. 2009. Design, Implementation and Evaluation of a Novel Public Display for Pedestrian Navigation: The Rotating Compass. In Proceedings of the SIGCHI Conference on Human Factors in Computing Systems (Boston, MA, USA) (CHI '09). Association for Computing Machinery, New York, NY, USA, 113-122. https://doi.org/10.1145/1518701.1518722

[15] Barbara Schehl and Jörg Leukel. 2020. Associations between individual factors, environmental factors, and outdoor independence in older adults. European fournal of Ageing 17 (2020), 291-298. https://doi.org/10.1007/s10433-020-00553-y

[16] Laura Stojko, Julian Fietkau, and Michael Koch. 2020. Design Guidelines for Micro Information Radiators to increase Seniors' Safety in Urban Space. In Mensch und Computer 2020 - Tagungsband (Magdeburg, Germany), Florian Alt, Stefan Schneegass, and Eva Hornecker (Eds.). Association for Computing Machinery, New York, NY, USA, 443-447. https://doi.org/10.1145/3404983.3410001

[17] Anselm Strauss and Juliet M. Corbin. 1990. Basics of qualitative research: Grounded theory procedures and techniques. Sage Publications, Inc.

[18] Erica Twardzik, Philippa Clarke, Suzanne Judd, and Natalie Colabianchi. 2020 Neighborhood Participation Is Less Likely among Older Adults with Sidewalk Problems. Fournal of Aging and Health 33, 1-2 (2020), 101-113. https://doi.org/ $10.1177 / 0898264320960966$
[19] Susanne Wallrafen, Jörg Leukel, and Barbara Schehl. 2017. Ergebnisse der Befragung von Seniorinnen und Senioren der Stadtteile Hardterbroich und Rheindahlen. https://www.urbanlifeplus.de/2017/09/ergebnisse-derbuergerbefragung-jetzt-online/ 